\title{
Self-Affine Elastic Contacts: Percolation and Leakage
}

\author{
Wolf B. Dapp, ${ }^{1}$ Andreas Lücke, ${ }^{1,2}$ Bo N. J. Persson, ${ }^{3}$ and Martin H. Müser ${ }^{1,4, *}$ \\ ${ }^{1}$ Jülich Supercomputing Centre, Institute for Advanced Simulation, FZ Jülich, Jülich, Germany \\ ${ }^{2}$ Department Physik, Universität Paderborn, Paderborn, Germany \\ ${ }^{3}$ Peter Grünberg Institute, FZ Jülich, Jülich, Germany \\ ${ }^{4}$ Department of Materials Science and Engineering, Universität des Saarlandes, Saarbrücken, Germany
}

(Received 27 January 2012; published 15 June 2012)

\begin{abstract}
We study fluid flow at the interfaces between elastic solids with randomly rough, self-affine surfaces. We show by numerical simulation that elastic deformation lowers the relative contact area at which contact patches percolate in comparison to traditional approaches to seals. Elastic deformation also suppresses leakage through contacts even far away from the percolation threshold. Reliable estimates for leakage can be obtained by combining Persson's contact mechanics theory with a slightly modified version of Bruggeman's effective-medium solution of the Reynolds equation.
\end{abstract}

PACS numbers: $46.55 .+\mathrm{d}, 83.50 . \mathrm{Ha}$

A seal is a device for closing a gap or making a joint fluid-tight [1]. Although seals play a crucial role in many modern engineering devices, inexpensive elastomeric seals such as O-rings are often used. The failure of seals can have serious ramifications ranging from energy loss, environmental pollution, expensive and time-consuming replacement procedures all the way to catastrophies like the Challenger disaster. Thus, seal systems should be handled thoroughly in the design of machines, and not like a secondary accessory.

Predicting leak rates is difficult, because the surface roughness at the seal-substrate interface spans a wide range of length scales, from nanometers to centimeters [2]. For accurate leakage calculations, one first needs to identify the gap topography and then solve the Reynolds thin-film equation, in which the local conductance is assumed to scale with the third power of the gap. Despite significant progress in the recent past [2-10], a comparison between large-scale numerical simulations (free of uncontrolled approximations) and approximate treatments is needed.

Industrial norms characterizing seal systems and traditional approaches to derive the gap or gap distribution function use as input the cumulative height distribution function of the free, undeformed surfaces also known as bearing area or Abbott and Firestone curve [1,11]. For example, gaps are constructed by simply "cutting" through the interface, i.e., $d(x, y) \equiv \max \left\{0, d_{\text {free }}(x, y)-\Delta d\right\}$, where $d_{\text {free }}(x, y)$ is the gap for nontouching surfaces as a function of the lateral coordinates $x$ and $y$, and $\Delta d$ is a constant shift.

These "bearing contacts" disregard that material in the vicinity of a contact point is being pushed away from the interface (elastic deformation). However, neglecting elastic deformation induces serious artifacts in contact mechanics. Relevant to seals are erroneous dependences of mean gap [12] and relative contact area [13] on normal load, as well as incorrect exponents for the contact autocorrelation functions [14], indicating flawed contact geometries. The contact geometry is crucial for the leakage problem, because stochastic indicators (such as the Euler characteristic [15]) in addition to the relative contact area $A / A_{0}$ (where $A$ is the true and $A_{0}$ the nominal contact area) determine whether insulating contact patches or open channels percolate. Therefore, approximations to the Reynolds thin-film equation that no longer contain information on the spatial arrangement of contact, such as Bruggeman's approach [16,17], may jeopardize the results.

A promising approach to the leakage problem $[2,6,7,9,10]$ is based on the contact mechanics theory by Persson [18], which was developed for the friction between rubber and hard randomly rough surfaces. The starting point is the analysis of how contact pressure or gap distribution functions broaden (on average for given surface height spectra) when finer and finer details of the surface topography are included in the calculation. The approach reduces a high-dimensional partial differential equation for the surface displacement to ordinary differential equations for pressure or gap distribution functions. Unlike traditional contact mechanics theories, Persson theory produces correct functional dependencies for the mean gap $[12,19,20]$ and relative contact [13] on load as well as exact exponents for the contact autocorrelation function $[21,22]$. Lastly, Persson theory corresponds to a rigorous expansion of an exact formulation of contact mechanics to at least third order in the inverse interfacial interaction range [23].

When applied to leakage, Persson theory needs to be complemented with approximate solvers to Reynolds' equation. One approach is to use Bruggeman's effectivemedium theory [16], which takes the gap distribution function as an input and predicts the percolation threshold to lie at a relative contact area of $A^{*} / A_{0}=1 / 2$.

Although Persson theory has predicted leakage and gap distribution functions in good agreement with both experiment [8,9,24] and numerical approaches [25], some fundamental issues remain to be addressed. First, the assumptions employed suffer to a certain degree from 
uncontrollable uncertainties, i.e., the precision of roughness spectra of the free surfaces, role of shear thinning where flow gradients are large, and the exact slip boundary conditions. The good agreement between theory and experiment may thus be partially fortuitous. Conversely, cancellation of errors can be noticed in simulations that realize all quantities to a defined precision. Second, it is not clear where the percolation threshold is, how it is affected by elastic deformation, and how previous approaches need to be altered, should $A^{*} / A_{0}$ deviate from the "canonical" value of $1 / 2$ [26]. Persson et al. found unexpectedly small values for $A^{*} / A_{0}$ in numerical simulations, but attributed this observation to finite size effects in simulation cells of a linear size of $\mathcal{L}=512$ grid points [17]. Other simulations $[27,28]$ also hint at the possibility that elastic contacts may percolate below $A / A_{0}=1 / 2$.

In this Letter, we produce "realistic" gaps by solving the elasticity equations for two rough solids in contact and solve the Reynolds equation for the produced gaps without uncontrolled approximations. We use $\mathcal{L}=4096$, which is large enough to reflect the self-affinity of the surface topography and also ensure self-averaging of the fluid conductance. This way we obtain percolation thresholds and leakage rates that are sufficiently accurate to determine the goodness of Bruggeman theory in elastic contacts and if $A^{*} / A_{0}$ deviates from $1 / 2$.

To solve the elastic problem, we use a slightly altered version of the Green's function molecular dynamics (GFMD) method presented in Ref. [29]. First, we reduce the displacement field to a scalar, thereby implicitly implementing the small-slope approximation [30]. Second, as Ref. [20], we use the continuum expression for the elastic energy, i.e., $V_{\mathrm{el}}=\sum_{\mathbf{q}} E^{*} q|\tilde{z}(\mathbf{q})|^{2} / 4$, where $\mathbf{q}$ is an in-plane wave vector, $q$ its magnitude, $E^{*}$ the effective elastic modulus, and $\tilde{z}(\mathbf{q})$ the Fourier transform of the normal displacement. Third, we solve Newton's equations of motion in Fourier space but implement the nonholonomic, hard-wall boundary conditions in real space. Fourth, we damp the modes such that the slowest mode is critically damped. This way the relaxation time scales with $\sqrt{\mathcal{L}}$. We map both compliance and roughness to one side of the interface, as is allowed for our system [30]. The substrate topography is generated in Fourier space as described in Ref. [21]; the height of the substrate satisfies the rules for colored noise of self-affine fractals, i.e., $\left\langle\tilde{h}^{*}\left(\mathbf{q}^{\prime}\right) \tilde{h}(\mathbf{q})\right\rangle \propto$ $\delta_{\mathbf{q q}} / q^{2+2 H}$, where $H$ is the Hurst roughness exponent. As a default, we allow for roughness between short and long wavelengths cutoffs of $\lambda_{s}=1$, and $\lambda_{l}=\mathcal{L} / 8=512$, respectively, but vary both bounds to reduce the risks of drawing false conclusions.

The gap topography produced in the GFMD simulation is used as the boundary condition for the Reynolds equation, which we solve with a central-differencing real-space method [31]. In order to speed up the calculations, we implemented a multigrid preconditioner; i.e., we first solve the Reynolds equation on a coarse grid, where the conductivity on each point is determined by invoking Bruggeman theory on the subpoints. The solution to the pressure on the coarse mesh is then interpolated onto a finer grid on which the lattice constant is halved. This way, the initial guess for the fluid pressure is already 3 orders of magnitude more accurate than the mean field solution when we reach the finest resolution.

We first address percolation on continuous random domains [26]. Given that the crossing of "coastlines" between contact and noncontact patches has zero measure in two dimensions, either contact or noncontact must percolate (except at the percolation threshold where stripes can occur). If the stochastic properties of contact at $A / A_{0}$ are identical to those of noncontact at $1-A / A_{0}$ (as is the case for bearing contacts of colored-noise surfaces), the percolation threshold must lie at $A^{*} / A_{0}=1 / 2$. We recover this value in our calculations, except for small scatter due to finite size. Discretization effects are minor in our calculations, because the contact correlation length distinctly exceeds a lattice constant, in particular, for our default roughness exponent $H=0.8$. This differs from conventional lattice models where adjacent grid points are uncorrelated, which makes $A^{*} / A_{0}$ depend on the lattice (simple cubic, hexagonal, etc.) and on the percolation type (bond versus site percolation) [32].

Including elastic deformation breaks the symmetry for the stochastic properties of contact and noncontact patches, as one can see in Fig. 1. Noncontact now tends to break up into many small lubrication pockets, while the contact patches tend to form connected areas with holes similar to Swiss cheese. In the language of algebraic topology, contact has a negative Euler characteristic and thus percolates more easily than noncontact with a positive Euler characteristic [15]. Because of the symmetry breaking of the stochastic properties for contact and noncontact patches, elastic contacts have their percolation threshold at $A^{*} / A_{0}<1 / 2$.

As passing comments we note that contact is defined as zero gap between the two surfaces. We verified that any finite separation leads to vanishing forces between the GFMD layer and its counterface. Furthermore, we find the same ratio of real contact area and load as in continuum treatments [33,34], despite our choice $\lambda_{s}=1$, because our elastic energy expression is that of a continuous rather than a discrete system.

Because of finite system size, a precise determination of $A^{*} / A_{0}$ remains difficult. We estimate $A^{*} / A_{0}$ by taking the average value of $A / A_{0}$ where contact and noncontact start to percolate throughout the system, respectively. Owing to some remaining discretization effects, the width of the transition region where no "color" unambiguously dominates is $\Delta A / A_{0}(\mathcal{L}=4096) \approx \pm 0.02$. The parameters considered in our study encompass: $H=0.4$ and $H=0.8$, $1 \leq \lambda_{s} \leq 4$, and $512 \leq \lambda_{l} \leq 2048$, as well as some disorder averaging over statistically equivalent surfaces 

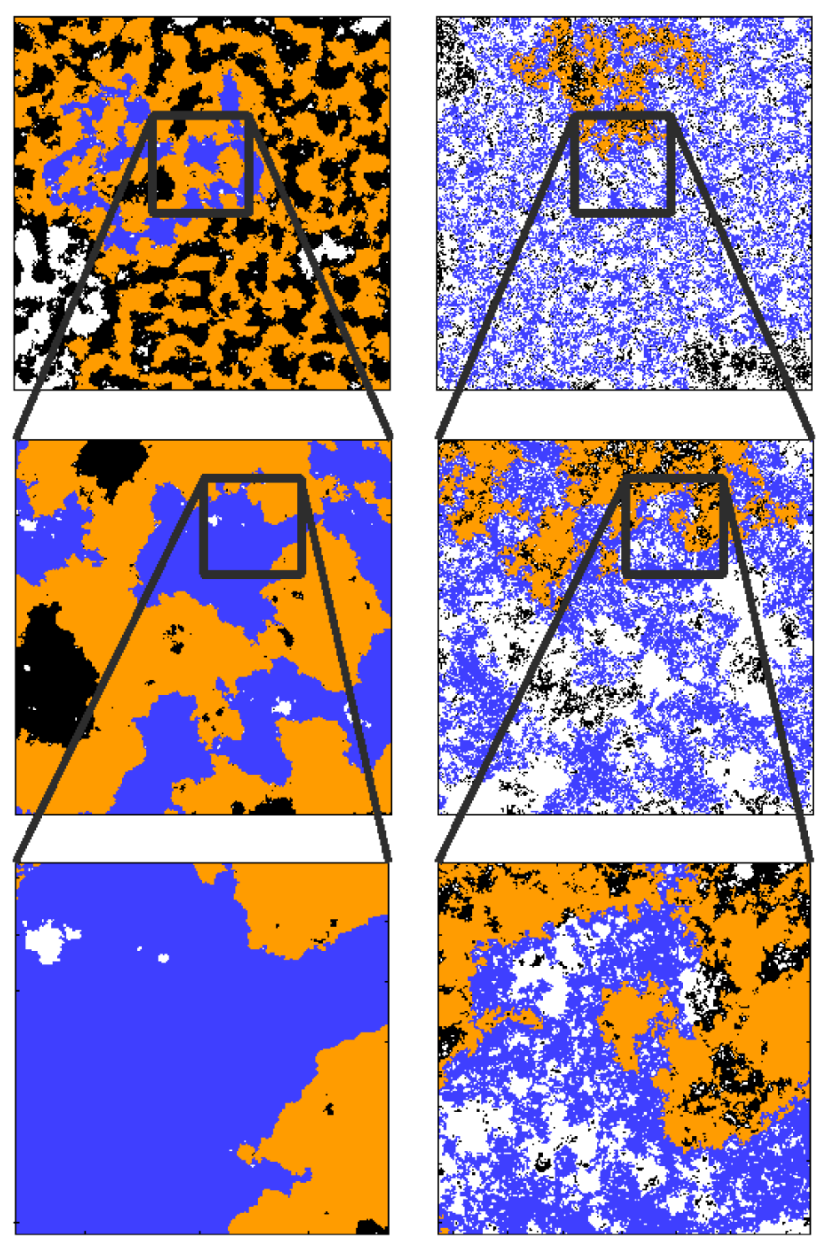

FIG. 1 (color online). Contact and noncontact patches for $A / A_{0}=0.46$ and $H=0.8$. Black is regular contact, while dark gray (blue) represents the largest connected contact patch. White and light gray (orange) represent similarly noncontact or open channels. Top panels show the full interface. Left: bearingarea model. Right: elastic calculations.

(using different random seeds). In all cases, we find $A^{*} / A_{0}=0.5 \pm 0.02$ for bearing contacts. This value is always reduced by $0.075 \pm 0.015$ when the contact is elastic, which leads us to an estimate of $A^{*} / A_{0}=$ $0.42(5)$. This is clearly less than the canonical value of $1 / 2$ and at most weakly dependent on $H$.

Since $A^{*} / A_{0}$ is smaller for elastic contacts one should expect reduced flow compared with bearing contacts. Indeed, the local maximum current intensities are reduced by three decades. Although the topography of the channel structure in elastic contacts resembles those of bearing contacts, Fig. 2 shows the channels to be much narrower for elastic contact, even far away from percolation.

We produced both elastic and bearing contacts for a variety of loads. Both types of contact topographies, more precisely their gap structures, were treated within the Bruggeman approximation [17]. We obtained full solutions to Reynolds' equation only for a few loads, as those are very time consuming due to their slow convergence.
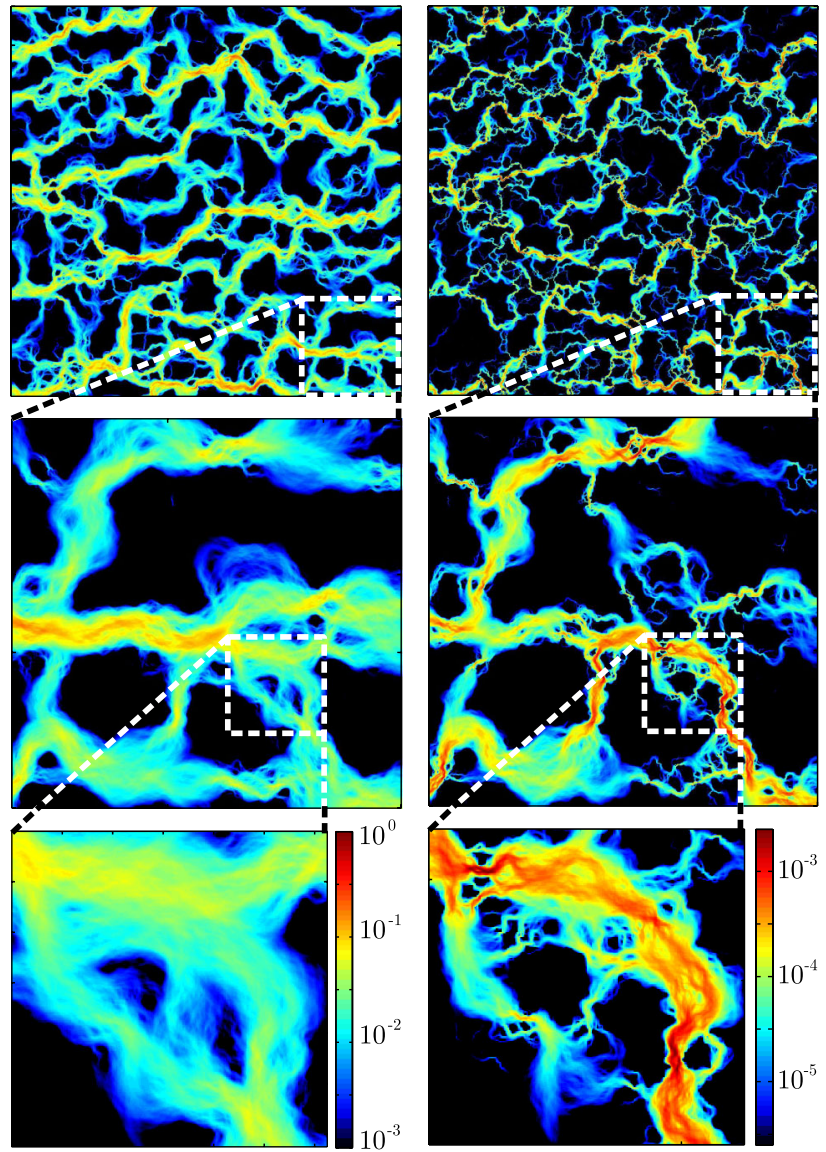

FIG. 2 (color online). Flow density through a contact with $A / A_{0}=0.2$. Left: bearing-area model. Right: elastic calculations. Note that the two scales differ by three decades. While generally the same channels are open, the flow is more constricted in the elastic case. Top panels show the full interface.

The results, shown in Fig. 3, demonstrate that current is strongly suppressed even far away from percolation. Furthermore, we find that the original Bruggeman theory is very accurate for bearing contacts. This is not surprising because Bruggeman theory is exact up to second order in the gap fluctuation [17] and also produces the exact percolation threshold for bearing contacts with colored-noise surface topographies.

For the elastic contact, we applied a small modification. First, we note that the Bruggeman effective-medium theory in $n$-dimensional space predicts that the noncontact area (for surfaces with roughness having isotropic statistical properties) percolates when $A^{*} / A_{0}=(n-1) / n$, e.g., $A^{*} / A_{0}=1 / 2$ for $n=2$. In the self-consistent equation for the conductivity, we replaced the physical dimension $n=2$ with an effective dimension $n_{\text {eff }}(A)$. For small contact area $A$, we want $n_{\text {eff }}$ to be close to the physical dimension of the interface, i.e., $n_{\text {eff }}(0)=2$, because Bruggeman is essentially exact where $A \ll A_{0}$. However, in order to move the percolation to the correct location, we need $n_{\text {eff }}\left(A^{*}\right)=1 /\left(1-A^{*} / A_{0}\right)$, i.e., for $A^{*}=0.42(5) A_{0}$, 


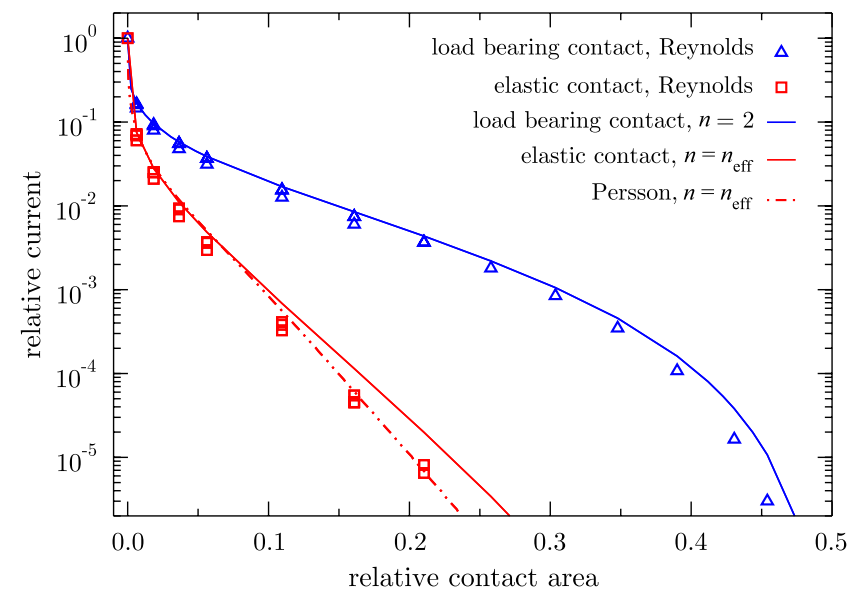

FIG. 3 (color online). Fluid current normalized by the value at first contact as a function of relative contact area. Symbols represent data from numerical solutions of the Reynolds equation for the bearing-area and elastic deformation models. Multiple symbols show surface topographies produced with different random seeds, and represent stochastic error. Solid lines are predictions using the modified Bruggeman theory. The dot-dashed line is the prediction using Persson's contact mechanics theory and the modified Bruggeman theory.

$n_{\text {eff }}\left(A^{*}\right)=1.72$. In between these two extremes we interpolate linearly. This is unnecessary for bearing-area surfaces, because they already have the Bruggeman threshold $A^{*} / A_{0}=1 / 2$. While our modification lessens some of the beauty of the original approach, Fig. 3 shows that it results in a very good agreement with the numerical solutions over several decades in the conductivity. The even better agreement achieved with Persson theory is owed to an $\mathcal{O}(10 \%)$ underestimation of the gap, which counteracts the overestimation of leakage in the Bruggeman theory.

To summarize, we have studied fluid flow at the interfaces between elastic solids with randomly rough surfaces and show by numerical simulation that elastic deformation lowers the relative contact area at which contact patches percolate [from 0.5 to $\approx 0.42(5)$ ], and suppresses leakage through such contacts even far away from the percolation threshold, in comparison to traditional approaches to seals. Leakage can be reliably estimated by combining Persson's contact mechanics theory with a slightly modified version of Bruggeman's effective-medium solution of the Reynolds equation.

We thank C. Denniston, N. Prodanov, and M. O. Robbins for useful discussions and the Jülich Supercomputing Centre for computing time.

*martin.mueser@mx.uni-saarland.de

[1] R. Flitney, Seals and Sealing Handbook (Elsevier, New York, 2007).

[2] B. N. J. Persson, O. Albohr, U. Tartaglino, A. I. Volokitin, and E. Tosatti, J. Phys. Condens. Matter 17, R1 (2005).
[3] F. Bottiglione, G. Carbone, L. Mangialardi, and G. Mantriota, J. Appl. Phys. 106, 104902 (2009).

[4] F. Sahlin, R. Larsson, A. Almqvist, P. M. Lugt, and P. Marklund, Proc. Instn. Mech. Engrs. Part J 224, 335 (2010).

[5] F. Sahlin, R. Larsson, P. Marklund, A. Almqvist, and P. M. Lugt, Proc. Instn. Mech. Engrs. Part J 224, 353 (2010).

[6] B. N. J. Persson, O. Albohr, C. Creton, and V. Peveri, J. Chem. Phys. 120, 8779 (2004).

[7] B. N. J. Persson and C. Yang, J. Phys. Condens. Matter 20, 315011 (2008).

[8] B. Lorenz and B. N. J. Persson, Europhys. Lett. 86, 44006 (2009).

[9] B. Lorenz and B. N. J. Persson, Eur. Phys. J. E 31, 159 (2010).

[10] B. N. J. Persson, J. Phys. Condens. Matter 22, 265004 (2010).

[11] E. J. Abbott and F. A. Firestone, Mech. Eng. Soc. Mech. Eng. 55, 569 (1933).

[12] B. N. J. Persson, Phys. Rev. Lett. 99, 125502 (2007).

[13] G. Carbone and F. Bottiglione, J. Mech. Phys. Solids 56, 2555 (2008).

[14] S. B. Ramisetti, C. Campañá, G. Anciaux, J.-F. Molinari, M. H. Müser, and M. O. Robbins, J. Phys. Condens. Matter 23, 215004 (2011).

[15] J. Schmähling and F. A. Hamprecht, Wear 262, 1360 (2007).

[16] D. A. G. Bruggeman, Ann. Phys. (Leipzig) 416, 636 (1935).

[17] B. N. J. Persson, N. Prodanov, B. A. Krick, N. Rodriguez, N. Mulakaluri, W. G. Sawyer, and P. Mangiagalli, Eur. Phys. J. E 35, 5 (2012).

[18] B. N. J. Persson, J. Chem. Phys. 115, 3840 (2001).

[19] B. Lorenz and B. N. J. Persson, J. Phys. Condens. Matter 21, 015003 (2009).

[20] S. Akarapu, T. Sharp, and M. O. Robbins, Phys. Rev. Lett. 106, 204301 (2011).

[21] C. Campañá, M. H. Müser, and M. O. Robbins, J. Phys. Condens. Matter 20, 354013 (2008).

[22] B. N. J. Persson, J. Phys. Condens. Matter 20, 312001 (2008).

[23] M. H. Müser, Phys. Rev. Lett. 100, 055504 (2008).

[24] B. Lorenz and B. N. J. Persson, Eur. Phys. J. E 32, 281 (2010).

[25] A. Almqvist, C. Campañá, N. Prodanov, and B. N. J. Persson, J. Mech. Phys. Solids 59, 2355 (2011).

[26] R. Zallen and H. Scher, Phys. Rev. B 4, 4471 (1971).

[27] G. Carbone (private communication).

[28] F. Sahlin, Ph.D. thesis, Luleå University of Technology, 2008; see, in particular, appendix F.

[29] C. Campañá and M.H. Müser, Phys. Rev. B 74, 075420 (2006).

[30] K. L. Johnson, Contact Mechanics (Cambridge University Press, , 1985).

[31] W. H. Press, S. Teukolsky, W. T. Vetterling, and B.P. Flannery, Numerical Recipes (Cambridge University Press, Cambridge, England, 2007).

[32] D. Stauffer and A. Aharony, Introduction to Percolation Theory (Taylor \& Francis, London, 1994).

[33] S. Hyun, L. Pei, J.-F. Molinari, and M. O. Robbins, Phys. Rev. E 70, 026117 (2004).

[34] C. Campañá and M. H. Müser, Europhys. Lett. 77, 38005 (2007). 